\title{
Decline in peripheral blood NKG2D+CD3+CD56+ NKT cells in metastatic colorectal cancer patients
}

\author{
Gharagozloo $\mathrm{M}^{1}$, Rezaei $\mathrm{A}^{1}$, Kalantari $\mathrm{H}^{2}$, Bahador $\mathrm{A}^{3}$, Hassannejad N${ }^{4}$, Maracy $\mathrm{M}^{5}$, Nouri $\mathrm{N}^{6}$, \\ Sedghi $\mathrm{M}^{6}$, Ghazanfari $\mathrm{H}^{1}$, Bayat $\mathrm{B}^{7}$
}

Department of Immunology, School of Medicine, Isfahan University of Medical Sciences, Isfahan, Iran. hd.ghazanfari@gmail.com

\begin{abstract}
OBJECTIVE: Colorectal cancer (CRC) is one of the main causes of cancer deaths in the world. This cancer can be divided into non-metastatic and metastatic CRC stages. CD3+CD56+ NKT cell subsets are a minor T cell subset in peripheral blood and conduct the killing of tumor cells in direct manner. Little is obvious about levels and surface markers of these cells such as NKG2D in different cancers, especially in CRC.

METHODS: We included 15 non-metastatic (low-grade), 11 non-metastatic (high-grade), 10 metastatic colorectal cancer patients and 18 healthy controls. The percentages of CD3+CD56+ NKT cells and NKG2D+CD56+ NKT cells from samples were analyzed by flow cytometry in peripheral blood mononuclear cells (PBMCs) of samples. RESULTS: We found that there was a significantly lower number of NKG2D+CD3+CD56+ cells in peripheral blood of patients with metastatic colorectal cancer compared with normal controls $(77.53 \pm 5.79 \%$ vs $90.74 \pm$ $9.84 \% ; p<0.01)$.

CONCLUSION: The fact that frequency of NKG2D+CD56+ NKT cells was significantly lower in patients with metastatic colorectal cancer compared to healthy controls strengthens the hypothesis that NKT cells can play a substantial role in the protection against human colorectal cancer, and this opens up avenues for novel studies about elucidating the other aspects of tumor surveillance in CRC progression and immunotherapy (Tab. 2, Fig. 2, Ref. 46). Text in PDF www.elis.sk.

KEY WORDS: low-grade non-metastatic colorectal cancer, high-grade non-metastatic colorectal cancer, metastatic colorectal cancer, CD3+CD56+ NKT cells, NKG2D.
\end{abstract}

\section{Introduction}

Colorectal cancer (CRC) is one of the main causes of cancer deaths in the world, especially in the developed countries. Based on TNM (Tumor/node/metastasis) system, this cancer can be divided into non-metastatic (I-III) and metastatic (IV) CRC stages (1). Similar to other cancers, a tumor can be classified as being of low or high grade (2).

Natural killer T (NKT) cells are innate-like T lymphocytes with unique activation and effector properties (3). The main features of NKT cells are co-expression of NK cell markers and T cell molecules (especially pan T cell marker CD3), CD1d restriction

${ }^{1}$ Department of Immunology, School of Medicine, Isfahan University of Medical Sciences, Isfahan, Iran, ${ }^{2}$ Department of Gasteroenterology, School of Medicine, Isfahan University of Medical Sciences, Isfahan, Iran, ${ }^{3}$ Department of microbiology, Faculty of Medicine, Tehran University of Medical Sciences, Tehran, Iran, ${ }^{4}$ Department of Cellular and Molecular Biology; Faculty of Sciences, Science and Research Branch of Islamic Azad University, Tehran, Iran, ${ }^{5}$ Department of Biostatistics and Epidemiology, School of Public Health, Isfahan University of Medical Sciences, Isfahan, Iran, ${ }^{6}$ Genetic lab, Al-Zhohada hospital, Esfahan University of Medical Sciences, Esfahan, Iran, and ${ }^{7}$ Department of Microbiology, Faculty of Medicine, Iran University of Medical Sciences, Tehran, Iran

Address for correspondence: H. Ghazanfari, Department of Immunology, School of Medicine, Isfahan University of Medical Sciences, Isfahan, Iran Phone: +982188955810
$(4,5)$. Totally, NKT cells are divided into three subsets, namely type I NKT cells also called invariant (i) NKT cells, type II NKT cells, and type III NKT or NKT-like cells (6). Some experiments indicate that NKT cells generate antitumor activity in solid tumors and certain hematologic forms of cancer (7). Some of these reports suggest a protective role for iNKT and NKT-like cells compared to type II NKT cells showing to be majorly immunosuppressive $(8,9)$. In general, iNKT cells were diminished significantly in solid tumors, and the low frequencies of peripheral blood iNKT cells associate with poor prognosis in plenty of cancers (10-12).

CD3+CD56+ NKT-like cells include roughly 5 to $15 \%$ of the peripheral T-cell pool. Owing to repeated exposure to antigen, these cells gently increase in absolute number in older persons but are absent in cord blood (1).

NKG2D (Natural Killer Group 2D; CD314) was found to be preferentially expressed by human natural killer (NK) cells, CD8+ T cells, and some auto-reactive CD4+ T cells (12-14). In NK cells, NKG2D functions as the primary activating receptor, while in $\mathrm{T}$ cells it acts as a co-stimulatory receptor that reinforces TCR-mediated activation (15-17). Consequently, NKG2D/NKG2DLs system may represent an important activation to begin a strong immune response to malignant cells (18). The NKG2D ligands (NKG2DLs) consist of a diverse array of proteins that are structurally related to MHC class I. The main targets recognized by NKG2D are MICA/B (MHC class I related molecules). NKG2D 
ligands are extensively expressed on solid tumors and in some forms of leukemia (15). Hence, owing to the loss of these ligands in normal cells and their expression in some types of cell stress such as cancers, NKG2D is considered to be one of the molecules involved in immune surveillance, particularly in the early stage of tumor development (19-24). In other words, NKG2D and its ligands serve as a self-immunosensing system and alert for host immune defense in response to tumors (25).

The presence of MICs on many progressing neoplasms, including breast, lung, gastric, renal, colon, ovarian, and prostate carcinomas and melanomas, indicates that MIC expression on tumors could enhance the immune evasion (26). During cancer progression, the immune pressure on the tumor may end up in the selection of cells devoid of NKG2D ligands. Accordingly, in the affected persons, most primary tumors appear to express NKG2D ligands, whereas more advanced tumors and metastasis express low levels (27-29). This situation has been associated with the transacting effects of soluble MICs (sMICs) cleaved from solid tumors and leukemic cells by a tumor-related metalloproteinase (30-32), followed by down-regulation of NKG2D through promoting its internalization and lysosomal degradation on immune cells (30).

Also, NKG2D was expressed on the surface of a unique CD3+CD56+ NKT-like cell subset (12). CD3+CD56+ NKT-like cells are a minor $\mathrm{T}$ cell subset in the peripheral blood $(<10 \%)$ and are at maximum in the liver (up to $30 \%$ ) (33). Considerably, these cells conduct non-MHC-restricted and MHC-restricted lysis of tumor target cells in direct cell-killing manner and displayed robust antitumor activity in adoptive transfer investigations (3438). To our knowledge, data demonstrated in our study regarding the NKG2D expression state on systemic NKT-like cells and frequency of these cells in non-metastatic and metastatic cancer patients are still lacking.

\section{Materials and methods}

\section{Patients' and controls' samples}

Heparinized peripheral blood was obtained from 36 patients with colorectal cancer attending the gastroenterology clinic of Al-zahra, khanevadeh, and seid-al-shohada hospitals, affiliated to Esfahan University of Medical Sciences, as well as 18 age- and sex-matched healthy volunteers. Written consent was obtained from all patients and healthy controls, and the study was approved by the Ethical Committee of Esfahan University of Medical Sciences. The disease diagnosis was based on the presence of symptoms of constipation in recent 6 months, history of familial polyposis, anus bleeding and positive colonoscopy results in relation to nonmetastatic patients, and history of colorectal cancer with current tumor dissemination to other organs in metastatic patients. TNM system is applied to classify CRC in four stages (I, II, III, and IV). We used both WHO (low and high grades) and TNM systems for the grouping of patients as follows:

- Normal: healthy individuals

- Non-metastatic (low-grade): stages I \& II

- Non-metastatic (high-grade): stage III

- Metastatic (distant metastasis): stage IV
Also, there is a checklist of histopathologic features to improve the standard of colorectal cancer reporting:

A: Histologic type assessed based on WHO classification as follows:

- Adenocarcinoma

- Mucinous adenocarcinoma

- Signet-ring cell carcinoma

- Small-cell carcinoma

- Squamous cell carcinoma

- Adenosquamous carcinoma

- Medullary carcinoma

- Undifferentiated carcinoma (2)

\section{Peripheral blood mononuclear cell (PBMC) isolation}

The most common method for PBMC isolation, FicollHypaque PBMC separation, was applied. Briefly, fresh peripheral whole-blood samples with heparin coagulant were diluted in one-to-one ratio with PBS/2 \% FBS solution. Then, the diluted blood sample was laid gently on Ficoll-Hypaque layer (sigma, St Louis, MO, USA) and finally, PBMC was separated from this solution via density centrifugation at 2,800 rpm for $20 \mathrm{~min}$. The viability of isolated cells was more than $95 \%$ as assessed by trypan blue exclusion test.

\section{Flow cytometry}

PBMC staining for FACS analysis was done with an optimized amount of the following fluorochrome conjugated mAbs: CD3PerCp, CD56-FITC, NKG2D-PE, PerCp Mouse IgG1 $\kappa$ Isotype Control, FITC Mouse IgG2b $\kappa$ Isotype Control, and PE Mouse IgG1 $\kappa$ Isotype Control. All mAbs with exception of NKG2D-PE and PE Mouse Isotype control (eBioscience; San Diego, CA; USA) were purchased from BD Bioscience (San Jose, CA, USA). The isolated cell suspension was adjusted at $10^{5}-10^{6} \mathrm{cell} / \mathrm{s} / \mathrm{ml}$ and incubated with anti CD3, anti CD56 and anti NKG2D for $30 \mathrm{~min}$ at $4{ }^{\circ} \mathrm{C}$. Simultaneously, daily, we incubated a tube with isotype controls for these three color stains. Ultimately, the cells were fixed with $0.5 \%$ paraformaldehyde solution in case that evaluation is delayed. The three-color analysis on FACS caliber (BD) was used to demonstrate the NKG2D expression on CD56+CD3+ as NKT-like cells.

\section{Statistics}

Statistical analysis was performed using the SPSS19 statistical software. The results were evaluated by one-way ANOVA and Kruskal-Wallis tests. Probability values of less than 0.05 were regarded significant. The results presented in text, tables and figures represent mean standard deviation (SD).

\section{Results}

\section{Characteristics of patients and healthy donors}

Patients were classified either in non-metastatic (low-grade; $n$ $=15)$, and non-metastatic (high-grade; $\mathrm{n}=11$ ), or metastatic $(\mathrm{n}=$ 10) groups (Tab. 1). All patients were enrolled to our study before surgery or chemotherapy to minimize the negative effect of these factors on their immune status. 
Tab. 1. Patient and healthy donor characteristics.

\begin{tabular}{llccc}
\hline Feature & & Normal & $\begin{array}{c}\text { Non-metastatic } \\
\text { (low-grade) }\end{array}$ & $\begin{array}{c}\text { Non-metastatic } \\
\text { (high-grade) }\end{array}$ \\
\hline Number & - & 18 & 15 & 11 \\
\hline Sex & Female & 8 & 7 & 4 \\
& Male & 10 & 8 & 7 \\
\hline Age & Minimum & 29 & 40 & 43 \\
& Maximum & 65 & 71 & 71 \\
\hline
\end{tabular}

Tab. 2. Mean frequency of CD3+CD56+ and NKG2D+CD3+CD56+cells and MFI of NKG2D on these cells in study groups.

\begin{tabular}{|c|c|c|c|c|c|}
\hline$\%$ & Control & $\begin{array}{l}\text { Non- metastatic } \\
\text { (low-grade) }\end{array}$ & $\begin{array}{l}\text { Non-metastatic } \\
\text { (high-grade) }\end{array}$ & Metastatic & $\mathrm{p}$ \\
\hline CD3+CD56+ cells in lymphocytes & $5.32 \pm 3.98$ & $6.99 \pm 2.73$ & $5.29 \pm 3.06$ & $4.76 \pm 2.64$ & 0.3 \\
\hline MFI of CD56 on CD3+CD56+ cells & $52.36 \pm 14.96$ & $48.42 \pm 20.40$ & $40.27 \pm 19.36$ & $48.85 \pm 17.69$ & 0.38 \\
\hline $\mathrm{NKG} 2 \mathrm{D}+\mathrm{CD} 3+\mathrm{CD} 56+$ cells & $90.74 \pm 9.84$ & $86.31 \pm 9.64$ & $82.74 \pm 12.39$ & $77.53 \pm 5.79$ & 0.009 \\
\hline MFI of NKG2D on NKG2D+CD3+CD56+ cells & $50.36 \pm 8.75$ & $46.95 \pm 8.32$ & $41.56 \pm 7.99$ & $37.94 \pm 8.24$ & 0.002 \\
\hline
\end{tabular}

The results represent mean percent $( \pm \mathrm{SD})$ of the cells and MFI $( \pm \mathrm{SD})$ of NKG2D expression on the related cells.

In our study, 30 samples were from patients with adenocarcinoma, and 6 cases were classified as mucinous adenocarcinoma. Similarly to previous studies, in our study, other types of tumor were rare (2).

Frequency of $\mathrm{CD} 3+\mathrm{CD} 56+$ NKT-like cells in patients with colorectal cancer and normal subjects

Our results showed no statistically significant difference among metastatic or non-metastatic colorectal cancer patients and healthy controls.

Frequency of NKG2D+ NKT-like cells in the study groups

By three-color flow cytometry method, we found a significantly lower number of NKG2D+CD3+CD56+ cells in peripheral blood of metastatic colorectal cancer compared with normal con- trols $(77.53 \pm 5.79 \%$ vs $90.74 \pm 9.84 \%$; $<<0.01)$; nevertheless, no significant differences were observed between non-metastatic patients and healthy subjects (Tab. 2, Figs 1A and 2).

Mean fluorescence intensity (MFI) of NKG2D expression on peripheral blood CD3+CD56+ cells in the patients and normal groups

In agreement with the frequency of NKG2D+NKT-like cells, the MFI of NKG2D on these cells was significantly reduced in the metastatic patients in comparison to the normal group (37.94 \pm 8.24 vs $50.36 \pm 8.75 ; p<0.005)$. Also, it was diminished less intensely in the non-metastatic (high-grade) patients compared with healthy controls $(41.56 \pm 7.99$ vs $50.36 \pm 8.75 ; \mathrm{p}<0.05)$ (Tab. 2 , Figs $1 \mathrm{~B}$ and 2).
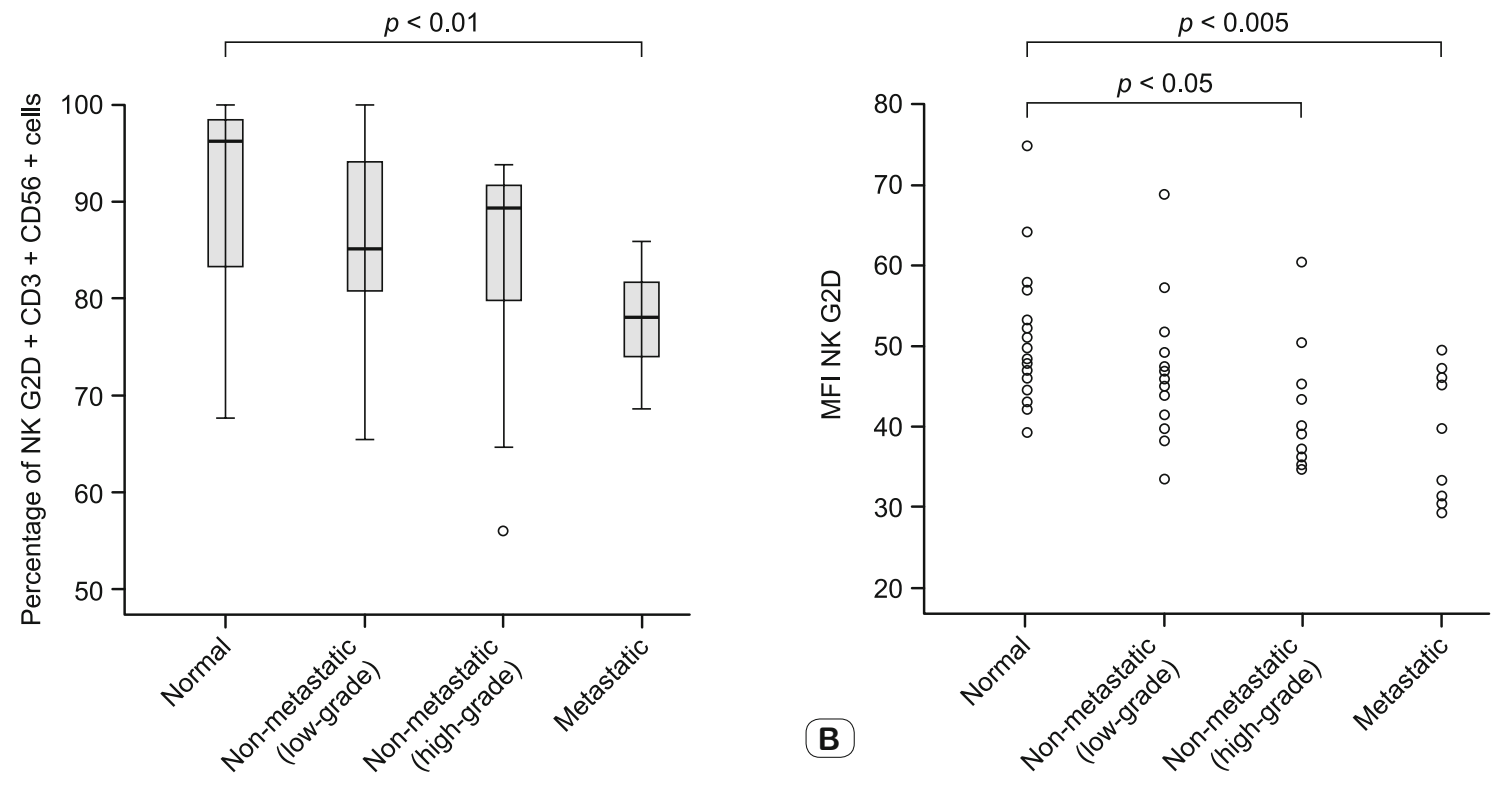

Fig. 1. A) Frequency (= percentage) of circulating NKG2D+ NKT-like cells, and B) Mean fluorescence intensity (MFI) of NKG2D expression on circulating NKT-like cells in the study patients vs normal controls. Significant $p$ ( $p<0.05$ or less) values are shown in both A \& B. 


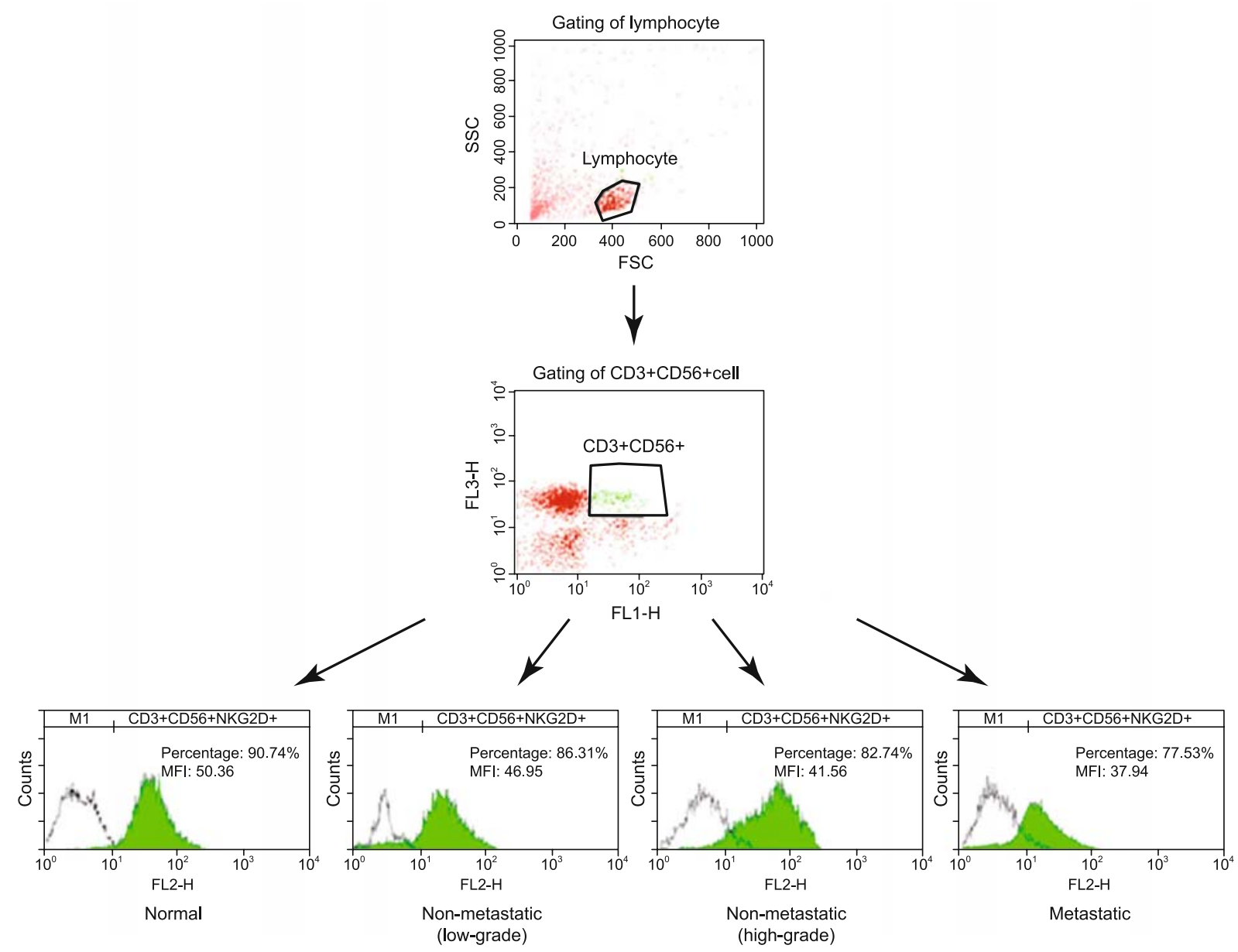

Fig. 2. Three-color flow cytometric analysis of NKG2D+CD3+CD56+ (NKG2D+ NKT-like) cells. First, lymphocytes gated in FSc (forward light scatter)-SSc (side light scatter) dot plot and then CD3+CD56+ gated in CD3-PerCp intensity vs. CD56-FITC intensity. Consequently FACS histograms show frequency (= percentage) of NKG2D+ NKT-like cells and MFI of NKG2D on these cells in the upper of each histogram. Colorless histograms represent isotype control antibodies and color histograms (green) show NKG2D as third color in FACS.

\section{Discussion}

Despite representing a small portion of systemic lymphocytes, CD3+CD56+ NKT-like cells show strong antitumor activities in vitro and in vivo through direct cell killing combined with other unique abilities to mediate immune cell recruitment and proliferation (39). In the study, we enumerated circulating CD3+CD56+ NKT-like cells; but the obtained data did not show any significant differences among frequencies of these cells in the study groups (Tab. 2). Besides NKT-like cells, a small proportion of Type I NKT (iNKT) cells are CD56+ $(40,41)$. The discrimination between these two (iNKT \& NKT-like) subsets requires the use of CD1d loaded with $\alpha$-GalCer ( $\alpha$-galactosylceramide) (42), lacking in our research. Thus, at this level, we could not determine the type of NKT cells in the patients and controls, and so we reported our results as CD3+CD56+ NKT cells.

Jadidi et al. showed that the amount of NKT-like cells in patients with CLL was significantly lower in comparison to healthy subjects and was inversely correlated with Treg cell proliferation and disease progression (43). It has already been demonstrated that Treg cells can suppress the proliferation, cytokine release and cytotoxic activity of NKT cells (44). However, in spite of increasing Treg cell levels in CRC, the role of these cells in CRC is currently under debate. For example, Blatner et.al displayed that in both human CRC and murine polyposis, the interaction between mast cell and Treg cell contributes to systemic regulatory $\mathrm{T}$ cell dysfunction, and switches from suppressing to generating potently immune suppressive, but proinflammatory Treg cells ( $\Delta$ Treg) (45) and this may be a convincing reason for our results about frequency of $\mathrm{CD} 3+\mathrm{CD} 56+\mathrm{NKT}$ cells contrary to the other tumor studies.

One of the important mechanisms employed by many cancers such as colon carcinoma, to promote immune evasion is the release of soluble forms of NKG2D ligands such as MICs, and consequential down-regulation of NKG2D expression with severe defects in cytotoxic activities of CD3+CD56+ NKT-like cells similar to NK and CTLs $(32,39,46)$. Regarding the lack of previous data about colorectal cancer, in this work, we indicated 
that the frequency of systemic CD3+CD56+ NKT cells and NKG2D expression on these cells decrease during the progression of colorectal cancer, especially in metastatic patients (Fig. 1 and Tab. 2). CD3+CD56+ NKT-like cells have been reported to induce NK-sensitive target U937 cell and NK-resistant Raji cell lysis in a non-MHC-restricted manner (2); whether NKG2D is engaged in the cell killing induced by CD3+CD56+ NKT-like cells remains unknown. The results of Wang et.al implied that the NKG2D receptor plays a fundamental role in cytolysis of ovarian and prostate cancer cells by CD3+CD56+ NKT-like cells, and this killing mediated by NKG2D is independent of TCR stimulation (39). The underlying mechanism has been associated with the trans-acting effects of sMICs cleaved from solid tumors and leukemic cells by a tumor-related metalloproteinase $(31,32)$. The engagement of sMICs then promotes NKG2D internalization and degradation in NK cells and CTLs. Although, we did not assess the circulating MICs levels in study groups to determine the correlation between ligands and lower NKG2D expression on CD56+NKT cells in colorectal cancer, there are some documents consistent with these findings that the induction or upregulation of MICs may promote tumor surveillance by engaging the activating receptor NKG2D on CD56+ NKT-like cells in leukemia patients and solid tumors. Also, there is evidence that the most important membrane-bounded NKG2DL ( MICA) was prevalent only in low-grade cancers and may be released into the tumor stroma and circulation in highgrade cancers as SMICA (soluble MICA) (46).

In conclusion, based on the fact that the frequency of NKG2D+CD56+ NKT cells and MFI of NKG2D expression on these cells were significantly lower in patients with metastatic colorectal cancer compared to healthy controls, it appears that sMICs may impact all NKG2D+ lymphocytes such as CD3+CD56+ NKT cells in the same manner, certainly in the advanced stages of cancer. These findings strengthen the hypothesis that NKT cells can play a substantial role in protection against human colorectal cancer and this opens up avenues of approach for novel studies about elucidating the other aspects of tumor surveillance in CRC progression and immunotherapy.

\section{References}

1. Thorsteinsson M, Jess P. The clinical significance of circulating tumor cells in non-metastatic colorectal cancer - a review. Eur J Surg Oncol 2011; 37 (6): 459-465.

2. Lanza G, Messerini L, Gafà R, Risio M. Colorectal tumors: the histology report. Dig Liver Dis 2011; 43 (11): 60590-60592.

3. Van Kaer L. NKT cells: T lymphocytes with innate effector functions. Curr opin immunol 2007; 19 (3): 354-364.

4. Godfrey DI, MacDonald HR, Kronenberg M, Smyth MJ, Van Kaer L. NKT cells: what's in a name? Nature Rev Immunol 2004; 4 (3): 231-237.

5. Kronenberg M. Toward an understanding of NKT cell biology: progress and paradoxes. Annu Rev Immunol 2005; 26: 877-900.

6. Godfrey DI, Hammond KJL, Poulton LD, Smyth MJ, Baxter AG. NKT cells: facts, functions and fallacies. Immunol Today 2000; 21 (11): 573-583.
7. Terabe M, Berzofsky JA. The role of NKT cells in tumor immunity. Adv Cancer Res 2008; 101: 277-348.

8. Berzofsky JA, Terabe M. The contrasting roles of NKT cells in tumor immunity. Curr Mol Med 2009; 9 (6): 667-672.

9. Giroux M, Denis F. CD1d - unrestricted human NKT cells release chemokines upon Fas engagement. Blood 2005; 105 (2): 703-710.

10. Dhodapkar MV, Geller MD, Chang DH, Shimizu K, Fujii SI, Dhodapkar KM, Krasovsky J. A reversible defect in natural killer T cell function characterizes the progression of premalignant to malignant multiple myeloma. J Exp Med 2003; 197 (12): 1667-1676.

11. Tachibana T, Onodera H, Tsuruyama T, Mori A, Nagayama S, Hiai H, Imamura M. Increased intratumor Valpha24-positive natural killer T cells: a prognostic factor for primary colorectal carcinomas. Clin Cancer Res 2005 Oct 15; 11 (20): 7322-7327.

12. Verneris MR, Karami M, Baker J, Jayaswal A, Negrin RS. Role of NKG2D signaling in the cytotoxicity of activated and expanded CD8+ T cells. Blood 2004; 103 (8): 3065-3072.

13. Groh V, Brühl A, El-Gabalawy H, Nelson JL, Spies T. Stimulation of $\mathrm{T}$ cell autoreactivity by anomalous expression of NKG2D and its MIC ligands in rheumatoid arthritis. Proc Natl Acad Sci 2003; 100 (16): 9452-9457.

14. Jamieson AM, Diefenbach A, McMahon CW, Xiong N, Carlyle JR, Raulet DH. The role of the NKG2D immunoreceptor in immune cell activation and natural killing. Immunity 2002; 17 (1): 19-29.

15. Coudert JD, Held W (Eds). The role of the NKG2D receptor for tumor immunity. 2006; 16 (5): 333-343.

16. Eagle RA, Jafferji I, Barrow AD. Beyond stressed self: evidence for NKG2D ligand expression on healthy cells. Curr Immunol Rev 2009; 5 (1): 22-34.

17. Bottino C, Castriconi R, Moretta L, Moretta A. Cellular ligands of activating NK receptors. Trends Immunol 2005; 26 (4): 221-226.

18. Watson NF, Spendlove I, Madjd Z, McGilvray R, Green AR, Ellis IO, Scholefield JH, Durrant LG. Expression of the stressrelated MHC class I chain-related protein MICA is an indicator of good prognosis in colorectal cancer patients. Int J Cancer 2006; 118 (6): 1445-1452.

19. Su HC, Nguyen KB, Salazar-Mather TP, Ruzek MC, Dalod MY, Biron CA. NK cell functions restrain T cell responses during viral infections. Eur J Immunol 2001; 31 (10): 3048-3055.

20. Groh V, Smythe K, Dai Z, Spies T. Fas ligand-mediated paracrine $\mathrm{T}$ cell regulation by the receptor NKG2D in tumor immunity. Nature Immunol 2006; 7 (7): 755-762.

21. Coudert JD, Zimmer J, Tomasello E, Cebecauer M, Colonna M, Vivier E, Held W. Altered NKG2D function in NK cells induced by chronic exposure to NKG2D ligand-expressing tumor cells. Blood 2005; 106 (5): 1711-1717.

22. Cerwenka A, Baron JL, Lanier LL. Ectopic expression of retinoic acid early inducible-1 gene (RAE-1) permits natural killer cell-mediated rejection of a MHC class I-bearing tumor in vivo. Proc Natl Acad Sci 2001; 98 (20): 11521-11526.

23. Guerra N, Tan YX, Joncker NT, Choy A, Gallardo F, Xiong N, Knoblaugh S, Cado D, Greenberg NR, Raulet DH. NKG2D-deficient mice are defective in tumor surveillance in models of spontaneous malignancy. Immunity 2008; 28 (4): 571-580. 
24. Cerwenka A, Lanier L. NKG2D ligands: unconventional MHC class I-like molecules exploited by viruses and cancer. Tissue Antigens 2003; 61 (5): 335-343.

25. López-Larrea C, Suárez-Alvarez B, López-Soto A, López-Vázquez A, Gonzalez S. The NKG2D receptor: sensing stressed cells. Trends Mol Med 2008; 14 (4): 179-189.

26. Osaki T, Saito H, Yoshikawa T, Matsumoto S, Tatebe S, Tsujitani S, Ikeguchi M. Decreased NKG2D expression on CD8+ T cell is involved in immune evasion in patients with gastric cancer. Clin Cancer Res 2007; 13 (2): 382-387.

27. Vetter C, Lieb W, Bröcker E, Becker J. Loss of nonclassical MHC molecules MIC-A/B expression during progression of uveal melanoma. Br J Cancer 2004; 91 (8): 1495-1499.

28. Raffaghello L, Prigione I, Airoldi I, Camoriano M, Morandi F, Bocca P, Gambini C, Ferrone S, Pistoia V. Mechanisms of immune evasion of human neuroblastoma. Cancer Lett 2005; 228 (1): 155-161.

29. Le Maux Chansac B, Moretta A, Vergnon I, Opolon P, Lécluse Y, Grunenwald D, Kubin M, Soria JC, Chouaib S, Mami-Chouaib F. NK cells infiltrating a MHC class I-deficient lung adenocarcinoma display impaired cytotoxic activity toward autologous tumor cells associated with altered NK cell-triggering receptors. J Immunol 2005; 175 (9): 5790.

30. Groh V, Wu J, Yee C, Spies T. Tumour-derived soluble MIC ligands impair expression of NKG2D and T-cell activation. Nature 2002; 419 (6908): 734-738.

31. Salih HR, Antropius H, Gieseke F, Lutz SZ, Kanz L, Rammensee HG, Steinle A. Functional expression and release of ligands for the activating immunoreceptor NKG2D in leukemia. Blood 2003; 102 (4): 1389-1396.

32. Doubrovina ES, Doubrovin MM, Vider E, Sisson RB, O’Reilly RJ, Dupont B, Vyas YM. Evasion from NK cell immunity by MHC class I chain-related molecules expressing colon adenocarcinoma. J Immunol 2003; 171 (12): 6891-6899.

33. Satoh M, Seki S, Hashimoto W, Ogasawara K, Kobayashi T, Kumagai K, Matsuno S, Takeda K. Cytotoxic gammadelta or alphabeta $T$ cells with a natural killer cell marker, CD56, induced from human peripheral blood lymphocytes by a combination of IL-12 and IL-2. T J Immunol 1996; 157 (9): 3886-3892.

34. Lu PH, Negrin RS. A novel population of expanded human $\mathrm{CD} 3+\mathrm{CD} 56+$ cells derived from $\mathrm{T}$ cells with potent in vivo antitumor activity in mice with severe combined immunodeficiency. J Immunol 1994; 153 (4): 1687-1696.

35. Baxevanis CN, Gritzapis AD, Papamichail M. In vivo antitumor activity of NKT cells activated by the combination of IL-12 and IL-18. J Immunol 2003 ; 171 (6): 2953-2959.
36. Saeterdal I, Straten PT, Myklebust JH, Kirkin AF, Gjertsen MK, Gaudernack G. Generation and characterization of GP-100 peptide-specific NK-T cell clones. Int J Cancer1998; 75 (5): 794-803.

37. Baxevanis CN, Gritzapis AD, Tsitsilonis OE, Katsoulas HL, Papamichail M. HER-2/neu-derived peptide epitopes are also recognized by cytotoxic CD3+ CD56+ (natural killer T) lymphocytes. Int J Cancer 2002; 98 (6): 864-872.

38. Wajchman HJ, Pierce CW, Varma VA, Issa MM, Petros J, Dombrowski KE. Ex vivo expansion of CD8+ CD56+ and CD8+ CD56-natural killer T cells specific for MUC1 mucin. Cancer Res 2004; 64 (3): 1171-1180.

39. Wang H, Yang D, Xu W, Wang Y, Ruan Z, Zhao T, Han J, Wu Y. Tumor-derived soluble MICs impair CD3 (+)CD56 (+) NKT-like cell cytotoxicity in cancer patients. Immunol Lett 2008; 120 (1-2): 65-71.

40. Hus I, Staroslawska E, Bojarska-Junak A, Dobrzynska-Rutkowska A, Surdacka A, Wdowiak P, Wasiak M, Kusz M, Twardosz A, Dmoszynska A, Rolinski J. CD3+/CD16+CD56+ cell numbers in peripheral blood are correlated with higher tumor burden in patients with diffuse large B-cell lymphoma. Folia Histochem Cytobiol 2011; 49 (1): 183-187.

41. Bojarska-Junak A, Hus I, Sieklucka M, Wasik-Szczepanek E, Mazurkiewicz T, Polak P, Dmoszynska A, Rolinski J. Natural killer-like T CD3+/CD16+ CD56+ cells in chronic lymphocytic leukemia: intracellular cytokine expression and relationship with clinical outcome. Oncol Rep 2010; 24 (3): 803-810.

42. Patel O, Cameron G, Pellicci DG, Liu Z, Byun HS, Beddoe T, McCluskey J, Franck RW, Castano AR, Harrak Y, Llebaria A, Bittman R, Porcelli SA, Godfrey DI, Rossjohn J. NKT TCR recognition of CD1d-alpha-C-galactosylceramide. J Immunol 2011; 187 (9): 4705-4713.

43. Jadidi-Niaragh F, Jeddi-Tehrani M, Ansaripour B, Razavi SM, Sharifian RA, Shokri F. Reduced frequency of NKT-like cells in patients with progressive chronic lymphocytic leukemia. Med Oncol 2012; 29 (5): 3561-3569.

44. Cava AL, Kaer LV. CD4+ CD25+ Tregs and NKT cells: regulators regulating regulators. TrendsIimmunol 2006; 27 (7): 322-327.

45. Blatner NR, Bonertz A, Beckhove P, Cheon EC, Krantz SB, Strouch M, Weitz J, Koch M, Halverson AL, Bentrem DJ. In colorectal cancer mast cells contribute to systemic regulatory T-cell dysfunction. Proc Natl Acad Sci 2010; 107 (14): 6430-6435.

46. Duan X, Deng L, Chen X, Lu Y, Zhang Q, Zhang K, Hu Y, Zeng J, Sun W. Clinical significance of the immunostimulatory MHC class I chain-related molecule A and NKG2D receptor on NK cells in pancreatic cancer. Med Oncol 2011; 28 (2): 466-474.

Received August 10, 2017. Accepted August 16, 2017. 